\title{
How do clinical staff in a large metropolitan hospital respond to disclosures of family violence?
}

\author{
Nadine Rudd ${ }^{1}$, Karen Willis ${ }^{2}$, Kim Felmingham ${ }^{3}$, Toni D Withiel ${ }^{4}$ and Caroline A Fisher, ${ }^{4 *}$ \\ ${ }^{1}$ Clinical Psychology, University of Melbourne and Royal Melbourne Hospital, Melbourne, Victoria, Australia \\ ${ }^{2}$ La Trobe University, Melbourne, Victoria, Australia \\ ${ }^{3}$ University of Melbourne, Melbourne, Victoria, Australia \\ ${ }^{4}$ Allied Health, Royal Melbourne Hospital, Melbourne, Victoria, Australia \\ ${ }^{5}$ The Melbourne Clinic, Richmond, Victoria, Australia
}

\begin{abstract}
Objective: Family violence is a critical public health issue. Best practice guidelines advise that clinicians respond by providing both practical and emotional support, which reflects the desires of victim survivors. However, there is little research analysing clinicians' provision of emotional support to victim survivors. This study aims to explore clinicians' responses to family violence in a large Australian public hospital, specifically examining the provision of emotional support.

Procedures: An online survey of clinical staff from nursing, medical, and allied health was conducted. Free-text responses were analysed using qualitative content analysis. There were 534 survey respondents, 241 of which provided free-text data.

Results: Four types of responses were identified ranging from non-active to active: Passing, Information Provision, Collaborating and Supporting. Overall, few clinicians indicated that they would provide emotional support when a disclosure occurred, with the majority describing non-active responses.

Conclusions: Findings suggest that very few clinicians report providing emotional support when responding to family violence disclosures, contrary to best practice guidelines. Given the disparity between best practice guidelines and self-reported clinical practice, findings highlight a need for specific training in providing emotional support following disclosures of family violence.
\end{abstract}

\section{Introduction}

Family violence (FV) is a significant public health issue in Australia. The Victorian Family Violence Protection Act [1] defines FV as behaviour towards a family member that causes fear, including physical, sexual, emotional, and economic abuse, and threatening, coercive, or dominating behaviour. In 2017, Victoria Police attended over 75,000 family incidents, where women comprised almost $75 \%$ of reported victims [2]. The consequences of FV are widespread and include contributing to rates of homelessness [3], substantial financial burden [4], and significant burden of disease [5]. The changed social and economic circumstances during the COVID-19 pandemic has raised concern about a rise in FV [6] with health services urged to maintain assistance to victim survivors, despite the burdens placed on services due to the pandemic [7].

The World Health Organisation (WHO) recommends that clinicians enquire about FV when indicators of violence are present [8]. Current WHO guidelines recommend a multi-faceted response to FV, using the acronym LIVES. Clinicians should:

Listen to and believe victim survivors,

Inquire about their needs and concerns,

Validate their experience,

Enhance safety, and offer ongoing

Support [9].
From this perspective, effective responses include both practical and emotional support based on the person's needs.

\section{Emotional support}

The term emotional support refers to those supportive behaviours that actively respond to a victim survivor's emotional needs $[10,11]$. This includes, but is not limited to, active listening, asking about emotions and emotional needs, and providing validation and reassurance. Research indicates that, following disclosures of violence, victim survivors desire individualised, non-judgemental responses that consider the complexities of FV, acknowledge that they are not at fault, and allow them autonomy [12]. However, clinician responses in a UK study suggested that almost half of clinicians would not provide a validating or supporting statement to identified victim survivors [13]. This indicates inconsistencies between the approach recommended by WHO, victim survivor preferences, and actual clinical practice when responding to disclosures.

${ }^{\star}$ Correspondence to: Caroline A Fisher, Psychology Advisor and Family Safety Team Lead Allied Health, 4 North Building, Royal Melbourne Hospital, 300 Grattan St Parkville, Victoria 3052, Australia, E-mail: caroline.fisher2@mh.org.au

Key words: disclosure, domestic violence, emotional support, family violence, healthcare, hospital

Received: April 21, 2021; Accepted: May 07, 2021; Published: May 10, 2021 


\section{The role of public hospitals}

With victim survivors having high usage rates of medical care [14], front-line hospital clinicians have a key role in identifying and supporting victim survivors. In $2016-17,29 \%$ of all assault hospitalisations in Australia were reportedly due to FV [15]. These figures are likely to be conservative, as not all FV related assault hospitalisations are reported as such. FV related assaults are high risk, with many presentations including head/neck and brain injuries [15].

The 2016 report of the Victorian Government Royal Commission into Family Violence emphasised the need for services to respond to victim survivors' needs quickly and appropriately, highlighted the key role of hospitals in responding to victim survivors, and called for a whole-of-hospital model for responding to FV [16]. The Strengthening Hospital Responses to Family Violence (SHRFV) initiative [17], which is now being rolled out across Victorian hospitals, is a whole-of-hospital training and support approach which aims to improve clinicians' responses to FV.

\section{Family violence disclosures and clinician responses}

The way in which clinicians enquire about violence can influence whether victim survivors disclose, with perceived clinician confidence and comfort increasing the likelihood of disclosure [18]. Victim survivors do not commonly spontaneously disclose FV, and are more likely to disclose if asked directly by a healthcare worker [19]. However, rates of enquiry about FV in healthcare settings are suboptimal, with one study indicating that many clinical staff in a general hospital (69\%) never or rarely screen patients for $\mathrm{FV}$, although screening rates appear to be higher in dedicated child and maternal health services [20]. Clinicians reported barriers to FV enquiry include time limitations and lack of knowledge [20,21], with some clinicians believing enquiry is outside their role and beyond their competence $[22,23]$.

Initial responses by healthcare workers impact on disclosing victim survivors $[19,24]$. Some victim survivors have reported being discouraged from talking about the violence, either explicitly or indirectly - for example, clinicians not following up on disclosures $[18,24]$. While some public hospitals have protocols for identifying $\mathrm{FV}$, clinicians need to be competent and confident in responding to disclosures, as supportive and empathetic responses encourage victim survivors to talk further about their experiences [24].

Most clinicians believe that supporting victim survivors is important [25]. However, research into clinician responses to FV has identified inconsistency and inadequacy in clinical responses. A recent study with Australian clinicians indicated that most clinicians use a patient-led response with a focus on enhancing safety [26], although unhelpful responses, such as being advised to take a holiday, or the clinican offering to help only the perpetrator, have been reported [19]. Inconsistencies are found across clinical disciplines regarding who should enquire about and respond to disclosures of reproductive abuse [26]. Other Australian research has indicated limited clinician confidence in screening, supporting, and referring victim survivors [25]. Victim survivors desire responses that address their emotional needs, and WHO best practice guidelines support this. With clinicians' initial responses to FV disclosures pivotal in whether victim survivors seek further help, more needs to be known about how clinicians respond to disclosures of FV.

\section{Methods}

The data reported in this paper was collected as part of a broader study at a large metropolitan public hospital in Victoria, Australia $[20,21]$. The study aimed to determine levels of FV training, self- perceived knowledge and confidence, specific clinical skills, and barriers to working effectively in the area. Data were collected via an online survey. The present paper examines responses to the question Do you have knowledge of what to do if/when a patient/client discloses family violence? Those providing Yes or Somewhat responses were requested to give a free-text response describing their practice (please describe what you would do). For these free-text responses, qualitative content analysis was used to analyse, first, the presence of emotional support and, second, the differences in responses between professions.

\section{Participants}

Participants were eligible for the study if they were members of medical, nursing, or allied health professions at the health service. Eligible participants were invited via email to participate in an anonymous self-report survey over a six-week period. Respondents $(n$ = 534) included medical, nursing, and allied health staff, and a variety of professional subgroups were represented within each discipline (see Table 1). From the total sample, 241 (45.13\%) clinicians provided freetext responses describing how they would respond to a FV disclosure. Allied health staff were significantly more likely to provide free-text responses than medical and nursing staff, who were significantly more likely to not provide free-text responses $\left(\chi^{2}(2, n=534)=91.44, p<\right.$ $.001)$. There were no significant differences in likelihood of providing free-text responses based on years of professional experience $\left(\chi^{2}(3, n=\right.$ $534)=3.55, p=.31)$.

\section{Qualitative content analysis}

The free-text responses were analysed using qualitative content analysis [27]. All answers were extracted and compiled into one text, which was read and re-read for familiarity with the content. Meaning units (words/phrases) were identified and labelled using codes developed inductively and compiled into a codebook. To enhance the credibility of the research findings, the codebook was discussed and agreed upon by two researchers (authors 1 and 4). The codes were further explored to determine the presence of emotional support, and the specific types of emotional support indicated. For example, if a clinician stated that they would maintain a non-judgemental stance, or that they would explicitly label the described behaviour as FV, this was coded as emotional support.

Table 1. Profession and practice areas

\begin{tabular}{|l|l|c|}
\hline Profession & Subgroup / Practice area & n \\
\hline Medicine (n=67) & Emergency & 18 \\
\hline & Acute & 24 \\
\hline & Sub-acute & 1 \\
\hline & Rehabilitation & 4 \\
\hline & Outpatient & 9 \\
\hline Nursing (n=242) & Other & 64 \\
\hline & Emergency & 141 \\
\hline & Acute & 30 \\
\hline & Sub-acute & 7 \\
\hline Allied Health & Rehabilitation & 52 \\
\hline (n=218) & Physiotherapy & 42 \\
\hline & Social Work & 17 \\
\hline & Psychology & 37 \\
\hline & Occupational Therapy & 18 \\
\hline & Speech Pathology & 18 \\
\hline & Nutrition / Dietetics & 34 \\
\hline & Other* & \\
\hline *Includes Allied Health Assistant, Podiatry, Music therapy & \\
\hline
\end{tabular}

*Includes Allied Health Assistant, Podiatry, Music therapy,

Prosthetics \& Orthotics, and those where profession was not specified. 
Responses were then sorted into categories, ranging from the most active to least active interventions. This analysis took into account the presence of active practical support as well as active emotional support, the extent to which responses were patient-centred, relevant safety measures, and referrals to colleagues and external service providers. The categories were then analysed to determine the proportions of each profession that were represented in each category. In reporting the textbased findings below, the profession and professional subgroup or area of practice is indicated.

\section{Results}

We conceptualised the qualitative responses as a Hierarchy of Support to encapsulate the shifts from passive to active provision of support evident in the 237 valid free-text responses about responding to disclosures (four responses were deemed invalid because they did not answer the question). Four hierarchical categories were identified: "Passing," "Information Provision," "Collaborating," and "Supporting" (Figure 1). Each category is seen as building on the last, i.e. Collaborating responses may also include Referral and Information Provision. The Supporting category sits at the top of the hierarchy, as these responses are regarded as best matching the guidelines for responding to $\mathrm{FV}$ disclosures specified by WHO.

The Passing category comprised 112 responses $(46.47 \%$ of freetext responses, $20.97 \%$ of total sample). In this category, clinicians indicated that they would focus primarily on discussing the disclosure with colleagues and external service providers. The most common response in this category indicated a referral to social work, e.g. "Involve social work" (Nursing, Acute). Other responses mentioned passing the information to colleagues, e.g. "Advise clinical supervisor or manager; inform care coordinator" (Allied Health, Podiatry). The Passing category included $63.54 \%(n=61)$ of free-text responses from nursing staff, $39.81 \%(n=43)$ of free-text responses from allied health staff and $30.30 \%(n=10)$ of free-text responses from medical staff (Figure 2 for distribution of answers by discipline). The responses in this category focussed on the conversations clinicians would have with colleagues and other service providers, and generally did not mention the conversations clinicians would have with the victim survivor.

The Information Provision category comprised 35 responses (14.52\% of free-text responses, $6.55 \%$ of total sample) that focussed on providing information to the victim survivor. Some clinicians were specific in what information they would provide, e.g. "Offer

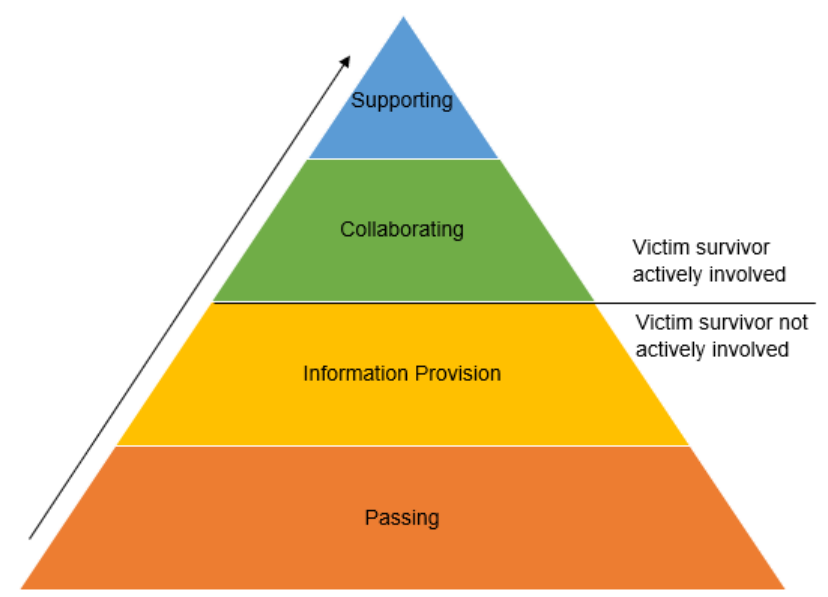

Figure 1. Hierarchy of disclosure support

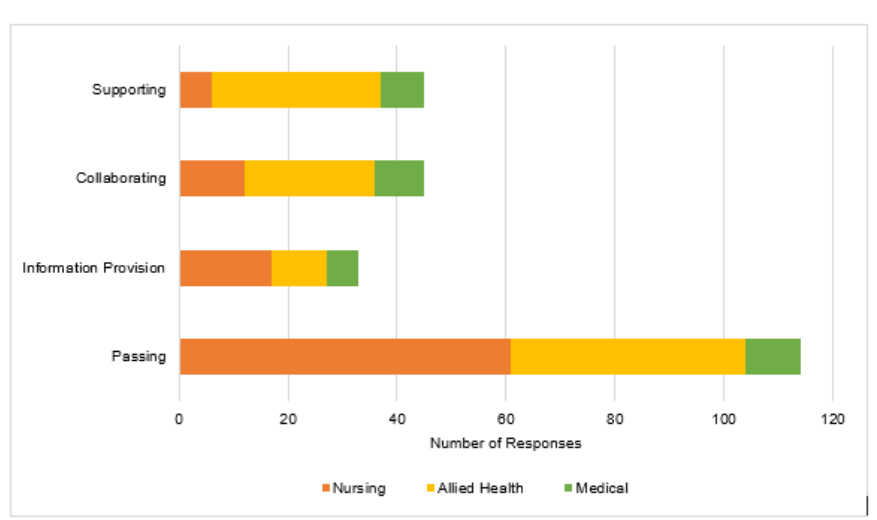

Figure 2. Hierarchy of support categories by discipline

some resources from the Domestic Violence Resource Centre in their local area" (Nursing, Acute), while others were non-specific, e.g. "Offer services" (Nursing, Emergency). In this category, clinicians mentioned providing information to victim survivors including contact numbers for external services, e.g. "Provide family violence support numbers ... Encourage patient to call police if they feel unsafe" (Allied Health, Psychology), and information regarding hospital-based support services, e.g. "Explain what the hospital may offer" (Medical, Rehabilitation). Responses of this type were provided by $18.18 \%(n=$ 6) medical staff who provided free-text responses, along with $17.71 \%$ ( $n=17)$ of nursing staff and $9.26 \%(n=10)$ of allied health staff. The responses in the Information Provision category may have included making a referral, but built on the responses in the Passing category by making explicit reference to the conversations they would have with the victim survivor. They tended to describe 'one-way' provision of information, rather than a dialogue between clinician and victim survivor; and it was the one-way provision of information that separated these responses from the next category in the hierarchy.

The 45 responses categorised as Collaborating (18.67\% of freetext responses, $8.43 \%$ of total sample) built upon the Information Provision responses by indicating that the clinician would collaborate with the victim survivor regarding further steps. These responses tended to describe patient-centred practice, including individualised risk assessments, asking victim survivors what they would like to happen, and seeking consent to speak with colleagues. Approximately one quarter of free-text responses $(27.27 \%, n=9)$ provided by medical staff were categorised as Collaborating, along with $22.22 \%(n=24)$ of responses by allied health staff and $12.50 \%(n=12)$ of responses by nursing staff. Examples in this category include:

"Listen to as many details as the person is comfortable sharing. Ask if they have any assistance/support. Ask consent to bring social work into the discussion" (Allied Health, Other);

"Would ask patient what supports they were after. I would then discuss with nurse in charge and/or social worker" (Nursing, Emergency).

The final category, Supporting, included 45 responses (18.67\% of free-text responses, $8.43 \%$ of total sample). Here, clinicians explicitly stated the practical or emotional support they would provide the victim survivor showing evidence of patient-centred practice. Most of these responses include explicit mention of emotional support (explored further in the next section). Just over one quarter $(28.70 \%, n=31)$ of free-text responses provided by allied health staff were categorised as Supporting, along with $24.24 \%(n=8)$ by medical staff and $6.25 \%(n=$ 6) by nursing staff. A typical example from this category is: 
"Explore with patient their experience, reflect their situation/ story, actively listen and support their story. Gently introduce options for management and support. Re-book patient for 1 week to review. Continue to build on supports available and explore what patient could see him/herself doing about this. Documentation, consent, supervision, advise team leader and manager, record on database, stat." (Allied Health, Social Work).

\section{Emotional support}

In total, 38 (15.77\% of free-text responses, $7.12 \%$ percent of overall sample) respondents stated that they would provide emotional support. All these responses fell in either the Collaborating or Supporting category ( 11 and 27 respectively). When clinicians mentioned emotional support, they most commonly wrote about listening, including active listening, e.g. "Listen to them, encourage them to talk..." (Allied Health, Social Work), "... Provide support, actively listen and offer assistance" (Nursing, Sub-Acute). Clinicians also wrote about validation, either explicitly, e.g. “... acknowledge, validate and engage patient..." (Allied Health, Social Work) or indirectly, e.g. "Acknowledge the impact on the patient and their family/carers" (Medical, Emergency).

Clinicians also mentioned explicitly labelling the described behaviour as FV, e.g. "Acknowledge that this IS family violence..." (Nursing, Acute). Similarly, some clinicians noted that they would emphasise to the victim survivor that FV is not acceptable, or that the violence is not their fault: "Reinforce that as the victim they are not responsible for the violence they are experiencing" (Allied Health, Physiotherapy).

In addition to indicating the language they would use, some clinicians stated that they would maintain a non-judgemental demeanour, e.g. "Listen, non-judgemental response..." (Allied Health, Social Work), "Express concern (hopefully without shaming the patient)" (Allied Health, Psychology). Other clinicians reported that they would believe the victim survivor, e.g. "Let them know you believe them..." (Allied Health, Social Work), "Believe them..." (Allied Health, Physiotherapy). Finally, several clinicians stated that they would thank the victim survivor for disclosing, e.g. "Thank them for having shared the story" (Nursing, Acute); "I would thank the patient for disclosing the information" (Allied Health, Occupational Therapy).

\section{Discussion}

Previous research has indicated that victim survivors disclosing FV desire a response that is non-judgemental and addresses their emotional needs. International best practice guidelines from the WHO reflect these findings and suggest that clinicians respond to both the practical and emotional needs of victim survivors. To date, there has been little research into whether clinicians are aware of the importance of emotional support, or whether clinicians provide emotional support in practice. This study aimed to address this gap in the literature, by examining clinicians' responses to disclosures of FV in a public hospital setting, specifically the provision of emotional support.

In our Hierarchy of Support, almost half of free-text responses were categorised as Passing; the category that encompassed the least active responses. Of the two thirds of respondents who indicated that they had at least some knowledge of what to do if they received a disclosure, around half of these reported that they would respond by discussing the disclosure with colleagues or making a referral. These clinicians did not report that they would provide any explicit support to, or have any specific conversations with, the victim survivor themselves. If we take these results as being indicative of the actual responses victim survivors will receive, the initial response to disclosures may fall short of a meaningful conversation or assessment.

Responses in the Information Provision category showed a more active role on the part of the clinician, by making some mention of communication between the clinician and the victim survivor. However, what separated these responses from those in the Collaborating category was the 'one-way' provision of information. Responses that included information being communicated from the victim survivor to the clinician were categorised as Collaborating, as the victim survivor was portrayed as playing a more active role in the interaction with the clinician. In this way, the four categories can be conceptualised as being inside two broader groups, with a division evident between Information Provision and Collaborating. This division provides an important separation, demarking clinicians who ask the victim survivor for consent, or how they wish to proceed, allowing the victim survivor to be more actively involved in their care, from those who do not. This division also marks the point at which the response to disclosures becomes patient-centred, as collaborating considers the individual needs of the victim survivor, as well as their wishes.

\section{Differences between disciplines}

Differences were noted between clinical disciplines. Responses from nursing staff were more likely to fall in the least active categories. Around two-thirds (63.54\%) of free-text responses from nursing staff fell in the Passing category and just $6.25 \%(n=6)$ of nurses providing a free-text response indicated they would actively provide emotional support. This contrasted with allied health and medical staff, where $39.81 \%$ of allied health staff who provided free-text responses and $30.30 \%$ of medical staff who provided free-text responses, provided responses in the Passing category. Conversely $4.30 \%$ of free-text responses from nursing staff fell in the Supporting category, compared with $28.70 \%$ of free-text responses from allied health staff and $24.24 \%$ of free-text responses from medical staff. While this may be a consequence of the open-ended nature of the question, the substantial difference between disciplines does suggest that when victim survivors disclose $\mathrm{FV}$, they may receive a different response depending on the discipline of the clinician to whom they disclose. Findings may reflect nursing staff beliefs that responding may be outside of their role within the hospital system, or their perception that there are barriers to them being able to respond to FV effectively. These differing responses between clinical disciplines are consistent with previous findings related to reproductive abuse [26].

\section{Emotional support}

Less than $20 \%$ of clinicians who provided a text response indicated that they would actively provide emotional support following a disclosure of FV. Taking the total sample into account (including those who did not provide a text response to this question, and/or indicated they did not know how to respond to a disclosure), only $7.12 \%$ of clinicians indicated they would provide emotional support. This represents significant missed opportunities to support victim survivors. As previously mentioned, hospital clinicians are uniquely positioned to respond to $\mathrm{FV}$, as hospitals are places victim survivors commonly present [14]. Moreover, victim survivors have indicated that having the violence acknowledged and receiving a non-judgemental, reassuring response encourages them to talk further about their experiences $[18,24]$. This highlights the importance of clinicians providing emotional support, validating the experience of victim survivors and maintaining a nonjudgemental approach. Interestingly, several clinicians in this study indicated they would thank the client for disclosing. This acknowledges 
the difficulty inherent in disclosing, that the clinician wants to know the information, and that it is not too confronting for the clinician.

Many clinicians (33.61\% of free-text responses, $n=81$ ) in this study indicated that their key response to disclosures would be to make a social work referral. It is understandable that some non-social work hospital clinicians may feel that providing relevant practical support (such as safety measures and external referrals) is outside their scope of practice or skill set. Ideally, however, every interaction between clinician and victim survivor should provide some level of support, be it practical, emotional, or both. If the clinician chooses a passive approach (e.g. makes a referral or puts an alert on the victim survivor's file) in isolation, their interaction with the victim survivor may not be actively addressing their needs. Basic emotional support, such as explicitly labelling the behaviour as FV or acknowledging the difficulty of disclosing, is one way that any clinician - from any discipline - can make their interaction with the victim survivor meaningful.

\section{Limitations}

The key limitation of these findings is the reliance on free-text data. Since the data is what clinicians have reported they believe they should do in response to a disclosure, it is difficult to make inferences about their actual practice. Similarly, the text-based nature of the data means that one cannot make strong inferences about the intent of clinicians, as researchers could not ask follow-up questions, and had to take the text at 'face value'. It is possible that those clinicians who did not report that they would provide emotional support do provide emotional support in practice, but do not consciously consider this as a clinical skill and so did not report it in their responses. Furthermore, not all clinicians provided free-text data for the question analysed here (even if they indicated that they knew how to respond to a disclosure, or knew somewhat), which limits the ability to generalise the present findings. Similarly, it is important to note that the proportions of clinical disciplines were skewed, with allied health staff overrepresented relative to their proportion of the hospital workforce, and medical staff underrepresented [20]. This also makes it difficult to generalise the findings pertaining to medical staff broadly. Despite these limitations, however, the data showed consistent patterns across the disciplines.

\section{Recommendations for training}

The results of this study lead to several implications for FV training in hospitals. First, the findings suggest that targeted training for individual disciplines will be more beneficial than generalist training. Second, these findings indicate a need for further training in the importance of emotional support, and the ways that clinicians from each discipline can provide emotional support. It may be useful for trainers to make a case for emotional support to clinicians by summarising the data on the importance of emotional support and the WHO guidelines for supporting victim survivors. Ideally, training will be interactive, and consider the extent of the clinicians' prior learning. For example, the SHRFV training modules include video examples of client engagement, written case vignettes, and examples of helpful versus unhelpful responses to disclosures [17]. Finally, the results indicate that FV training needs to include specific guidance on whose role it is to respond to FV - namely, that all hospital staff can (and indeed should) provide active practical and/or emotional support to victim survivors of FV.

\section{Conclusions}

FV is a significant contemporary public health issue in Australia. Hospitals have been recognised as significant sites for intervention to address the health effects of FV; this study reveals that there remains much to be done to ensure that disclosures of FV in this setting are responded to appropriately.

Our findings also have important implications for clinical practice. For clinicians working with victim survivors who have already made a disclosure of FV, these findings indicate that one cannot assume that the victim survivor has received emotional support or has been consulted about their desired outcome of the disclosure. Thus, it is important for all clinicians to provide emotional support. Accurate recording of the support provided (eg whether a referral to social work was made; or the type of information provided) is also vital. Further research is recommended focusing on the translation of training about FV into practice; for example, qualitative research where clinicians recall a time when they responded to a disclosure, may provide a more accurate representation of clinical responses. Such research may also build on the present findings of inconsistency across disciplines with a more indepth analysis of these differences.

\section{Acknowledgements}

The author team thanks the clinical staff of [name] hospital who contributed their time to participating in this research.

\section{Funding}

This research was supported by Strengthening Hospital Responses to Family Violence (SHRFV) funding awarded to the Royal Melbourne Hospital by the Victorian State Government.

\section{Competing Interests}

The author team have no competing interests to declare.

\section{References}

1. Family Violence Protection Act (2008) Victorian Current Acts, Australia.

2. Crime Statistics Agency (2018) Crime Statistics Victoria: Year Ending 31 December 2017

3. Australian Institute of Health and Welfare (AIHW) (2017) Specialist Homelessness Services Report 2016-17.

4. KPMG (2016) The Cost of Violence Against Women and their Children in Australia: Final Report. Canberra: Department of Social Services.

5. Ayre J, Lum On M, Webster K, Gourley M, Moon L (2016) Examination of the burden of disease of intimate partner violence against women in 2011: Final report. ANROWS Horizons p. 6.

6. Usher K, Bhuller N, Durkin J, Guyamfi N, Jackson D (2020) Family violence and COVID-19: Increased vulnerability and reduced options for support. Int J Ment Health Nurs 29: 549-552. [Crossref]

7. Roesch E, Amin A, Gupta J, Garcia-Moreno C (2020) Violence against women during covid-19 pandemic restrictions. BMJ 369: m1712. [Crossref]

8. World Health Organization (WHO) (2013) Responding to Intimate Partner Violence and Sexual Violence Against Women: WHO Clinical and Policy Guidelines.

9. World Health Organization (WHO) (2014) Health Care for Women Subjected to Intimate Partner Violence or Sexual Violence: A Clinical Handbook (Field Testing Version).

10. Wills TA, Shinar O (2000) Measuring perceived and received social support. Social Support Measurement and Intervention: A Guide for Health and Social Scientists, Oxford University Press 4: 86-135.

11. Burleson BR (2003) Emotional support skills. Handbook of Communication and Social Interaction Skills p: 551-594.

12. Feder GS, Hutson M, Ramsay J, Taket AR (2006) Women exposed to intimate partner violence: expectations and experiences when they encounter health care professionals: a meta-analysis of qualitative studies. Arch Intern Med 166: 22-37. [Crossref]

13. Ramsay J, Rutterford C, Gregory A, Dunne D, Eldridge S, et al. (2012) Domestic violence: knowledge, attitudes, and clinical practice of selected UK primary healthcare clinicians. Br J Gen Pract 62: e647-e655. [Crossref] 
14. Campbell JC (2002) Health consequences of intimate partner violence. Lancet 359: 1331-1336. [Crossref]

15. Australian Institute of Health and Welfare (AIHW) (2019) Family, Domestic and Sexual Violence in Australia: Continuing the National Story.

16. State of Victoria (2016) Royal Commission into Family Violence: Summary and Recommendations. Parl. Paper No. 132 (2014-16).

17. Strengthening Hospital Responses to Family Violence (SHRFV) (2018) Strengthening Hospital Responses to Family Violence Project Management Guide: A Practical Guide for Establishing and Implementing a Service Model.

18. Bacchus L, Mezey G, Bewley S (2002) Women's perceptions and experiences of routine enquiry for domestic violence in a maternity service. BJOG: Int J Obstet Gy 109: 9-16. [Crossref]

19. Bacchus L, Mezey G, Bewley S (2003) Experiences of seeking help from health professionals in a sample of women who experienced domestic violence. Health Soc Care Community 11: 10-18. [Crossref]

20. Fisher C, Rudkin N, Withiel T, May A, Barson E, Allen B, et al. (2020) Assisting patients experiencing family violence: A survey of training levels, perceived knowledge and confidence of clinical staff in a large metropolitan hospital. Women's Health 16: 1-10. [Crossref]

21. Withiel, T. D., Gill, H. \& Fisher, C. A (2021). Responding to family violence: Variations in knowledge, confidence and skills across clinical professions in a large tertiary public hospital. SAGE open medicine, 9, 20503121211000923.
22. Withiel T, Allen B, Evans K, Rudkin N, Willis K, Hooker L, et al. (2020) Assisting clients experiencing family violence: Clinician and client survey responses in a child and family health service. J Clin Nurs 29: 4076-4089. [Crossref]

23. Feder G, Ramsay J, Dunne D, Rose M, Arsene C, Norman R, et al. (2009) How far does screening women for domestic (partner) violence in different health-care settings meet criteria for a screening programme? Systematic reviews of nine UK National Screening Committee criteria. Health Technol Assess p. 13. [Crossref]

24. Rose D, Trevillion K, Woodall A, Morgan C, Feder G, Howard L (2011) Barriers and facilitators of disclosures of domestic violence by mental health service users: qualitative study. Br J Psychiatry 198: 189-194. [Crossref]

25. Trevillion K, Howard LM, Morgan C, Feder G, Woodall A, Rose D (2012) The response of mental health services to domestic violence: a qualitative study of service users' and professionals' experiences. J Am Psych Nurses Assoc 18: 326-336. [Crossref]

26. Soh HJ, Grigg J, Gurvich C, Gavrilidis E, Kulkarni J (2018) Family violence: An insight into perspectives and practices of Australian health practitioners. $J$ Interpers Violence 36: 5-6. [Crossref]

27. Tarzia L, Wellington M, Marino J, Hegarty K (2019) How do health practitioner in a large Australian public hospital identify and respond to reproductive abuse? A qualitative study. Aust NZ J Publ Heal, 43: 457-463.

28. Graneheim UH, Lundman B (2004) Qualitative content analysis in nursing research: concepts, procedures and measures to achieve trustworthiness. Nurs Educ Today 24 105-112. [Crossref]

Copyright: (C2021 Rudd N. This is an open-access article distributed under the terms of the Creative Commons Attribution License, which permits unrestricted use, distribution, and reproduction in any medium, provided the original author and source are credited. 